\title{
Simulations and real applications of PI and MPC averaging level control in a water resource recovery facility
}

\author{
Finn Aakre Haugen ${ }^{1}$ \\ ${ }^{1}$ University of South-Eastern Norway, Norway, finn.haugeneusn.no
}

\begin{abstract}
The basin level control system in an existing wastewater treatment plant is designed for two different operation modes: compliant level control for smooth pump flow, and stiff level control. Both a model-based predictive controller (MPC) and a standard PI controller are implemented, and tested both on a dynamic simulator and on the real plant. The simulator, the MPC and the PI controller tuning are based on a mathematical process model derived from a material balance of the wastewater in the inlet basin.
\end{abstract}

Keywords: Water resouce recovery facility, equalization magazine, level, MPC, Kalman Filter. PI control.

\section{Introduction}

This article reports results from a study where alternative controllers of the level of the equalization magazine upstream to the VEAS water resource recovery facility (wrrf) ${ }^{1}$ at Slemmestad, Norway has been tested both on a dynamic simulator and on the real plant. This magazine comprises the part of the inlet tunnel being closest to the wrrf. VEAS is the largest wrrf in Norway, serving about 700,000 population equivalents (pe), treating in average about $3.5 \mathrm{~m} 3 / \mathrm{s}$. The biological treatment at the wrrf will benefit from a smoother hydraulic load than at present. One approach to this end is improving the level control system of the inlet basin, aiming at smoother pump flow from the basin to the treatment processes (Bolmstedt, 2004); (van Overloop et al., 2010).

The implementation of the simulator and the control system used in this study is in LabVIEW (National Instruments) with the MPC algorithm implemented in MATLAB code in LabVIEW's MATLAB Script node. The sampling time (time-step) of the various discrete-time algorithms of simulation, estimation, filtering, and control is $10 \mathrm{~s}$.

The paper is organized in the following main sections: System description; Controller functions; Results; Conclusion; Abbreviations; Nomenclature; Acknowledgements; References.

\footnotetext{
1 "wrrf" is the terminology recommended by the International Water Association (IWA) and the Water Environment Federation (WEF)
}

\section{System description}

\subsection{Geometrical design}

Figure 1 depicts the principal geometrical design of the inlet part of the VEAS wrrf.

\subsection{Operation modes of the tunnel and basin and requirements to the level control sys- tem}

The tunnel and basin are operated in different modes:

- Operation mode \#1: Normally low load (tunnel flow) \& Compliant level control: The main aim of this mode is to obtain smooth pump flow. To this end, compliant level control is implemented: The level is allowed to vary between the soft limits of $1.5 \mathrm{~m}$ and $2.5 \mathrm{~m}$, with $1.8 \mathrm{~m}$ as the nominal level setpoint. Lately, these limits have been changed to $1.6 \mathrm{~m}$ and $2.8 \mathrm{~m}$, respectively, with setpoint $2.3 \mathrm{~m}$. (Both these sets of specifications are used in this article.)

- Operation mode \#2: Normally low load \& Stiff level control: The main aim of this mode is to have the level close to a relatively low setpoint to ensure that the pump soaks up solid downfall from the wastewater accumulated in the basin during Operation mode \#1. The duration of this operation mode is relatively short, approximately two hours, each second day. In this operation mode, the variations of the pump flow will, inevitably, be relatively large as they are almost the same as the variations of the net inflow to the basin.

In addition to Operation modes \#1 and \#2, there are operation modes concerning normally high load (at high preciptiation) and to tunnel flushing (building up a volume of wastewater in a part of the tunnel, and then flushing the tunnel with this volume). This article covers only Operation mode \#1.

\subsection{Mathematical model of the inlet basin}

The basis of both the simulator and the model-based predictive controller is a dynamic model of the liquid level of the basin, Eq. 1. The model stems from material balance assuming the sewage is water.

$$
\dot{h}(t)=\frac{F_{\text {in }}(t)-F_{\text {out }}(t)}{A(h)}
$$




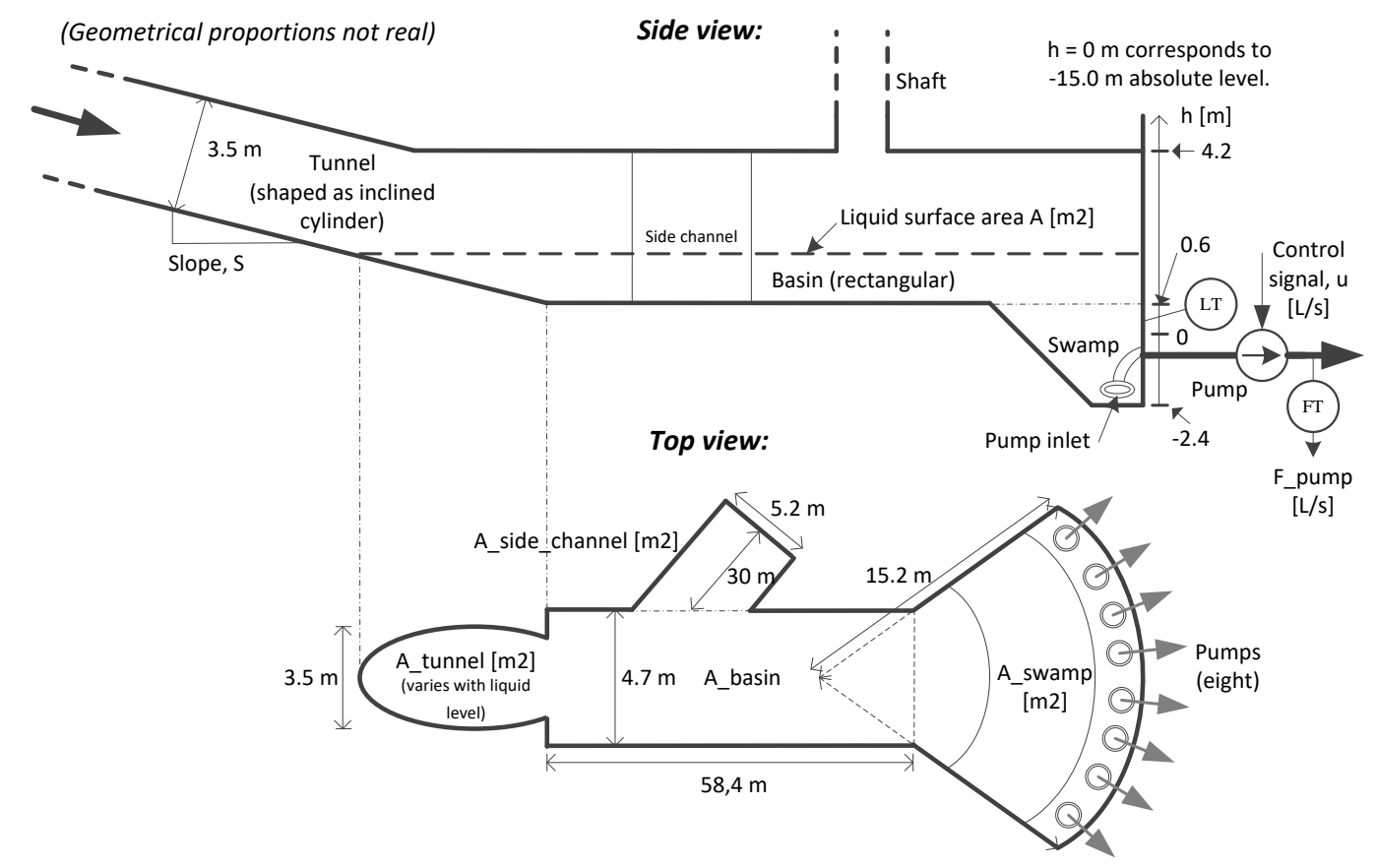

Figure 1. Principal design of inlet basin of VEAS wrrf.

where

$$
F_{\text {out }}(t)=F_{\text {pump }}(t)
$$

and

$$
F_{\text {in }}(t)=F_{\text {in }_{\text {meas }}}(t)+F_{\text {in }_{\text {nonmeas }}}(t)
$$

where $F_{\text {in }}$ are measured flows and $F_{\mathrm{in}_{\text {nonmeas }}}$ are unmeasured flows.

The liquid surface area $A$ in Eq. 1 is calculated from the assumed known geometry, and is therefore a function of the liquid level, $h$. The ellipsoidal liquid surface in the tunnel is continuously calculated by numerical integration. The dynamics of the pump is taken into account by the level controllers. In the real plant, an apparent time-delay of approximately $120 \mathrm{~s}$ is observed between the pump control signal $\mathrm{u}$ and the resulting (measured) pump flow $F_{\text {pump }}$ :

$$
F_{\text {pump }}(t)=u\left(t-T_{\text {delay }_{\text {pump }}}\right)
$$

For conservative controller tuning, the pump dynamics is represented by a time-delay of $T_{\text {delay }}$ pump $=120 \mathrm{~s}$.

\subsection{Piping and Instrumentation Diagram (P\&I diagram)}

Figure 2 shows a P\&I diagram of the level control system. The flow at Vækerø is measured. Vækerø is situated approximately $15 \mathrm{~km}$ upstreams the plant, The flow at Vækerø constitutes the main inflow component to the plant, counting for $70-80 \%$ of the total tunnel inflow. This flow arrives at the plant with a transportation time (timedelay) of approximately $3.0 \mathrm{~h}$, but smoothed, so the transportation time is not well-defined. The smoothing is represented with a time constant low pass filter with an estimated time constant of $1 \mathrm{~h}$. The Vækerø flow measurement is used in simulations. However, it is not used by the controllers in the real implementations in this study due to the uncertain information about its contribution to the actual inflow to the basin, as pointed out above.

\section{Controllers}

Two different controllers are used in this study, namely (a) PI control and (b) MPC.

\subsection{PI controller}

A standard PI controller (Seborg et al., 2004) is used:

$$
u(t)=K_{c} e(t)+\frac{K_{c}}{T_{i}} \int_{0}^{t} e(\theta) d \theta
$$

The PI controller is tuned with the Skogestad method (Skogestad, 2003) for "integrator plus time-delay" process dynamics, but with a modification where the integral time, $T_{i}$, is reduced to obtain faster disturbance compensation (Haugen and Lie, 2013). In general, the following differential equation can represent such dynamics:

$$
\dot{y}=K_{i} u(t-\tau)
$$

By neglecting $F_{\text {in }}$, the process model, Eqs. $1-4$, is on the form of Eq. 6 with

$$
\begin{gathered}
y=h \\
u=F_{\text {pump }} \\
K_{i}=-\frac{1}{A}
\end{gathered}
$$

The Skogestad PI settings for the model Eq. 6 are:

$$
K_{c}=\frac{1}{K_{i}\left(T_{c}+\tau\right)}=-\frac{A}{T_{c}+\tau}
$$




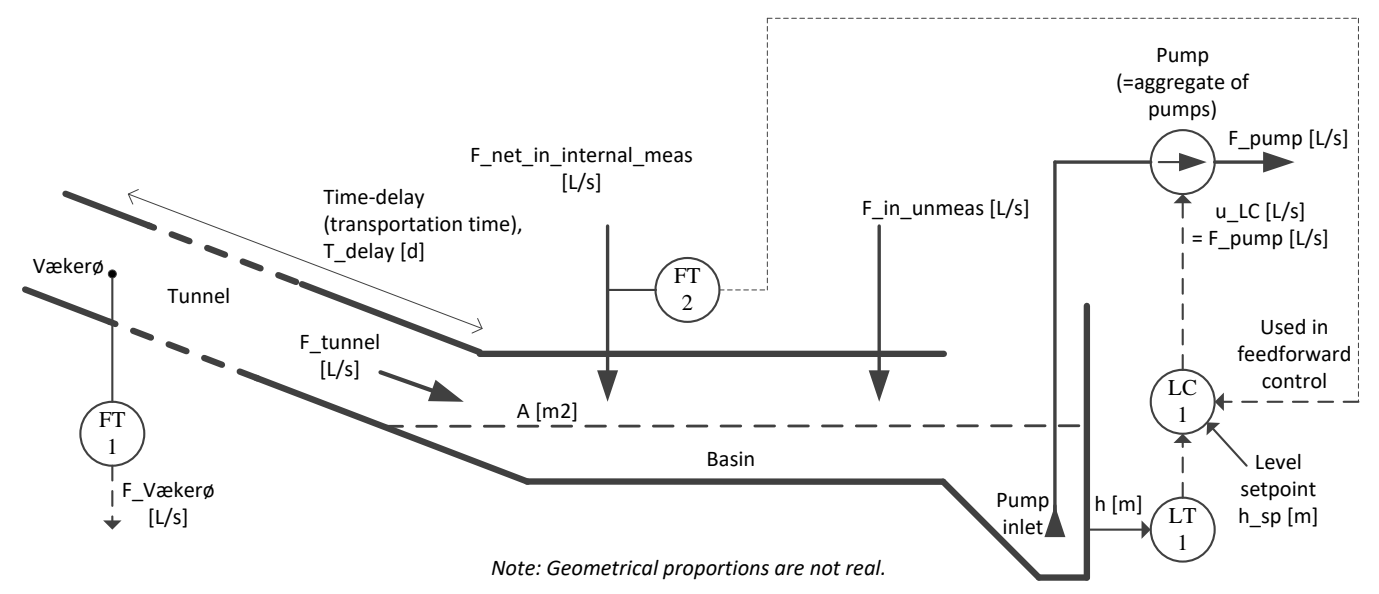

Figure 2. Piping and Instrumentation diagram (P\&I D) of level control system of basin.

$$
T_{i}=2\left(T_{c}+\tau\right)
$$

where $T_{c}$ is the closed (control) loop time constant specified by the user. In general, the PI controller can be tuned for satisfactory performance using $T_{c}$ as follows: By increasing $T_{c}$, the control systems typically becomes more sluggish: The control signal becomes smoother, and the control error becomes larger. On the other side, by decreasing the closed loop time-constant, the control systems typically becomes more aggressive (faster): The control signal varies more abruptly, and the control error becomes smaller.

\subsection{MPC with Kalman Filter}

A nonlinear MPC is used (Grüne and Pannek, 2011). The optimization (minimization) problem of the MPC used in this study is ${ }^{2}$

$$
\min _{u}\left[J=\int_{t_{0}}^{t_{0}+T_{p}} C_{1} e(t)^{2}+C_{2} \dot{u}(t)^{2} d t\right]
$$

The optimization or decision variable is the pump flow (control variable). $e=h_{\mathrm{sp}}-h$ is control error. $\dot{u}=\dot{F}_{\text {pump }}$ is rate of change of pump flow (control variable). $C_{1}$ and $C_{2}$ are cost coefficients. $T_{p}$ is the prediction horizon. $t_{0}$ is the present point of time. The MPC finds the sequence of sampled future pump flow values that gives the optimal balance or compromise between small control error and small rate of change of pump flow. To save the computional demand, control signal locking is used, i.e. the number of allowable values during the prediction horizion is set to $N_{p}$.

A time-constant filter with time constant $T$ pump was included in the calculation of the applied control signal to the pump to obtain improved pump flow smoothing.

The MPC uses a Kalman Filter estimate of the total unmeasured flows, see below.

\footnotetext{
${ }^{2}$ Although the objective function, $J$, is here on a continuous-time form, a corresponding discrete-time form is of course used in the computer implementation.
}

The MPC takes into account level constraints and control variable (pump flow) constraints. MATLAB's fmincon function is used as optimization function.

Kalman Filter. The estimate of the total of the unmeasured flows is calculated with an Extended Kalman Filter (EKF) (Simon, 2006) based on the process model, Eqs. 1 3 with total unmeasured flow, $F_{\text {in }_{\text {nonmeas }}}$ modelled as a "random walk" augmentation state variable. The time delay in Eq. 4 was neglected as it is assumed to have negligible effect on the estimates.

To summarize, the model used in the EKF comprises the following two differential equations:

$$
\begin{gathered}
\dot{h}=\frac{F_{\mathrm{in}_{\text {meas }}}+F_{\text {in }_{\text {nonmeas }}}-F_{\text {pump }}}{A(h)}+w_{1} \\
\dot{F}_{\text {in }_{\text {nonmeas }}}=0+w_{2}
\end{gathered}
$$

where $w_{1}$ and $w_{2}$ are random process disturbances.

The process output measurement used by the EKF to correct the predicted state estimate is the measured level.

The process measurement noise covariance was set to

$$
R=[0.1]
$$

and the process disturbance covariance was set to

$$
Q=\left[\begin{array}{cc}
0.1 & 0 \\
0 & 10^{-6}
\end{array}\right]
$$

In the tuning, $R$ and $Q(1,1)$ were fixed to more or less random values, and then $Q(2,2)$ was used as the ultimate tuning parameter since it directly affects the estimate of $F_{\text {in }_{\text {nonmeas }}} \cdot Q(2,2)$ was adjusted by trial-and-error to give a sufficiently fast while not too noisy flow estimate. $R$ might have been set to the variance of representative time-series of the level measurement, but this was not implemented. In practice, it is mainly the ratio between the variances that determines the behaviour of the estimator. 


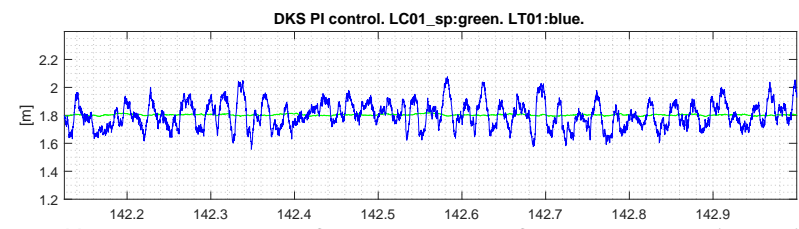

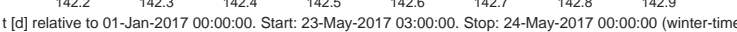

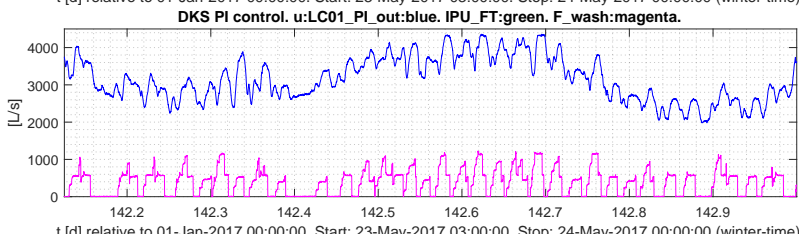

t $[$ d] relative to 01-Jan-2017 00:00:00. Start: 23-May-2017 03:00:00. Stop: 24-May-2017 00:00.
DKS PI control. du_dt:blue. du_dt_high_lim and du_dt_low_lim:red.

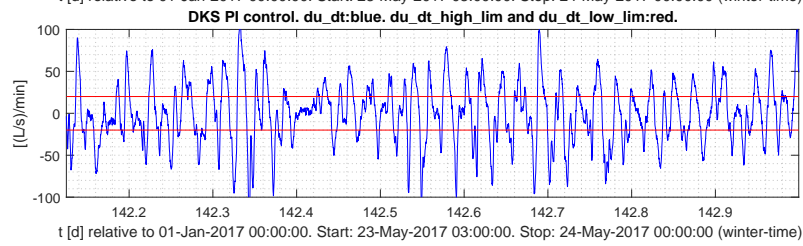

Figure 3. Results with real PI level control with original PI settings. (Time range: $21 \mathrm{~h}$.)

\section{Results}

\subsection{Real PI level control with original PI set- tings}

Figure 3 shows real responses with the following original PI controller settings (before the retuning presented in this article):

$$
K_{c}=-3200(\mathrm{~L} / \mathrm{s}) / \mathrm{m}, T_{i}=1000 \mathrm{~s}
$$

which have been found with trial and error. The level setpoint is $1.8 \mathrm{~m}$. The absolute value of the rate of change of the pump flow, $|\dot{u}|$, becomes substantially larger than the specified maxium of $20(\mathrm{~L} / \mathrm{s}) / \mathrm{min}$. The fraction of time that $|\dot{u}|$ is larger than $20(\mathrm{~L} / \mathrm{s}) / \mathrm{min}$ is $57 \%$.

\subsection{Simulated level control with retuned PI controller}

The simulator was driven by real flow measurements. The PI controller was tuned with the Skogestad method with $T_{c}=1000 \mathrm{~s}$ adjusted by trial and error on the simulator to satisfy the specifications. The resulting PI settings are:

$$
K_{c}=-2000(\mathrm{~L} / \mathrm{s}) / \mathrm{m}, T_{i}=2000 \mathrm{~s}
$$

The simulated responses are shown in Figure 3 (together with responses with MPC). The specification to the rate of change of pump flow is met, see the lower plot in Figure 4. Also, the level is within the limits.

\subsection{Simulated level control with MPC}

The MPC was tuned with $C_{1}=1$ (fixed) and $C_{2}=0.05$ by trial and error. The time step was $120 \mathrm{sec}$, and the prediction horizon was 30 minutes, corresponding to 15 time steps. Control signal blocking with $N_{p}=3$ blocks
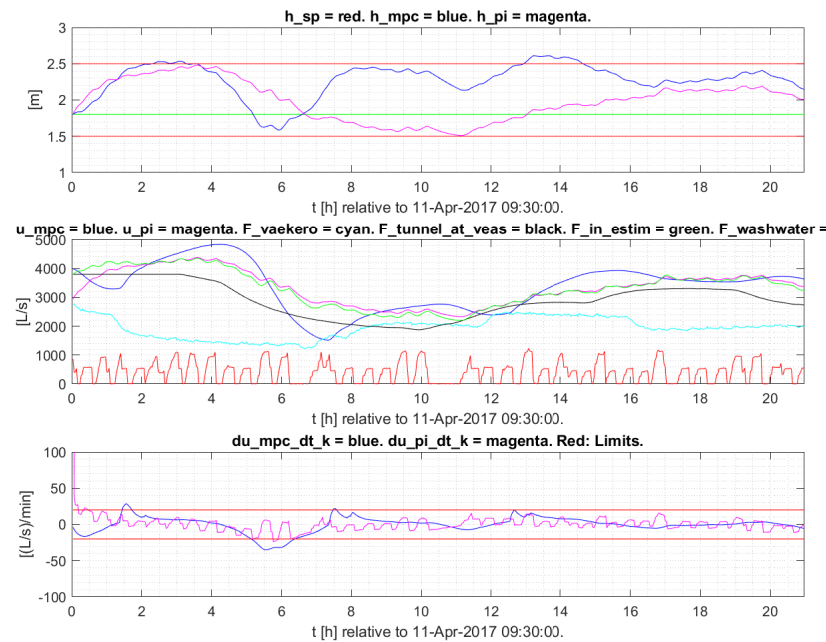

Figure 4. Results with simulated PI level control (magenta curves) and MPC level control (blue curves). (Time range: 21 h.)

of equal size (5 time steps) was implemented. The pump time-constant filter was set by trial and error to $T_{\text {pump }}=$ $700 \mathrm{~s}$.

The simulated responses are shown in Figure 4. The specification to the rate of change of pump flow is met except for a small time interval around time $t=6 \mathrm{~h}$, see the lower plot in Figure 4. The level is within the limits except for a small time interval around $t=14 \mathrm{~h}$.

\subsection{Real level control with retuned PI con- troller}

A retuned PI level controller was applied to the real plant. The retuning was based on the Skogestad method. It was decided to increase the normal level setpoint from originally $1.8 \mathrm{~m}$ to $2.3 \mathrm{~m}$. This level increase implies that the liquid surface area, A, increases, thereby reducing the process gain. The reduction of the process gain allows for a more relaxed PI tuning (larger $T_{c}$ ).

The Skogestad tuning was based on the specification $T_{c}=1500 \mathrm{~s}$. A time-delay of $T_{\text {delay }_{\text {pump }}}=120 \mathrm{~s}$ was included in the process model to account for pump control dynamics. The liquid surface area was fixed to $A=2000$ $\mathrm{m}^{2}$ in the tuning, which is approximately the area at the operating point of level $h=1.8 \mathrm{~m}$.

The resulting tuning became

$$
K_{c}=-1240(\mathrm{~L} / \mathrm{s}) / \mathrm{m}, T_{i}=3240 \mathrm{~s}
$$

Figure 5 shows the results on the real plant. As seen from the lower plot, the specifications to the maximum rate of the pump flow is satisfied.

The PI settings above are now (as of May 2018) in ordinary use at the plant. 


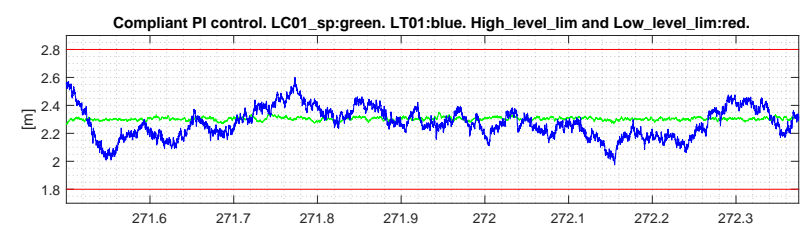

12:00:00. Stop: 30-Sep-2017 09:00:00 (winter-time)
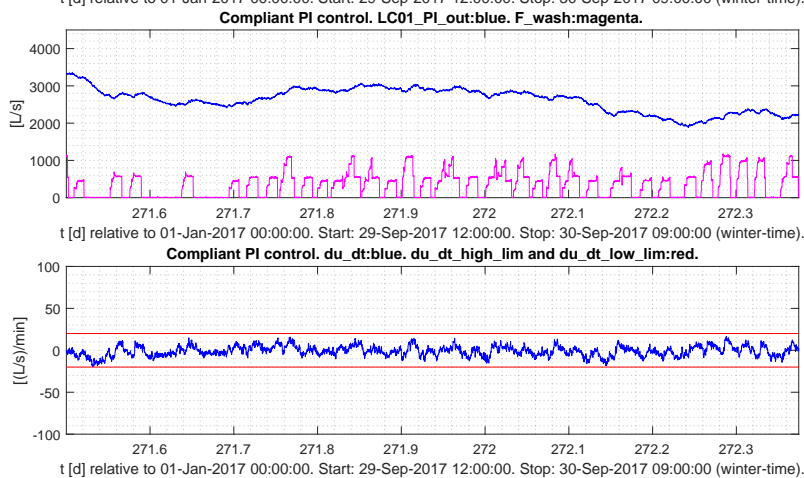

Figure 5. Results with PI control on the real plant. (Time range: $21 \mathrm{~h}$.

\subsection{Real level control with MPC}

Due to practical reasons, only initial results of MPC level control applied to the real plant can be shown in this article. Both a simulation study similar to the study presented above, and real tests were accomplished. Both these tests were accomplished without including the lowpass filter to smooth the pump flow. The MPC pump control signal showed abrupt changes at the points of time when the MPC decided to change the control signal, see Figure 6 . The control signal resembles a piecewise constant signal. This behaviour is actually understandable since $\dot{u}(t)$ is zero except at the short transitions, making the rate of change term, $\dot{u}(t)^{2}$, in the MPC objective relatively small.

Several solutions were tried to mitigate the abrupt control signal changes. The best solution turned out to be the pump lowpass filter, but from these tests, we only have simulation results, cf. Section 4.3. Unfortunately, the equipment was not longer available for a practical test which might have confirmed the good simulation results with the improved MPC.
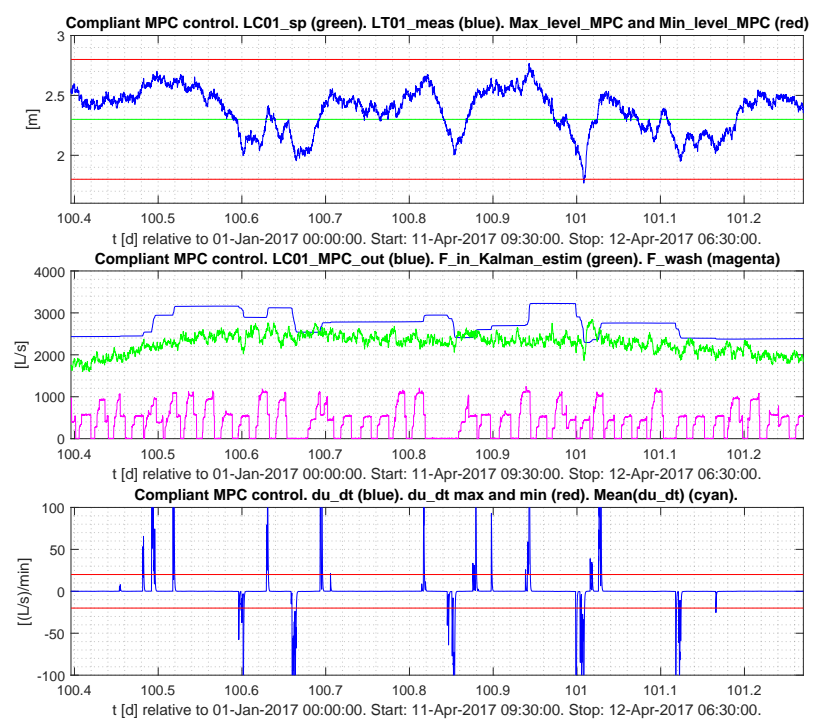

Figure 6. Results with MPC on the real plant. (Time range: 21 h.)

\section{Conclusions}

Successful averaging level control was obtained with a PI controller tuned with the Skogestad tuning method, both on the simulated plant and on the real plant.

The first implementation of MPC for averaging level control applied to the real plant was not successful as the pump flow pattern generated by the controller showed abrupt changes between the time intervals of piecewise constant flow. By including a lowpass filter on the control signal in the MPC model, successful control was eventually obtained with MPC - on the simulated plant. Unfortunately, due to practical reasons, this successful MPC implementation could not be tested on the real plant.

This study has demonstrated, to the author and to the staff at the plant, the power of using a dynamic simulator for tuning and testing control systems.

In the hindsight, one lesson to learn from this study is that enough time should be allocated to, hopefully, obtain satisfying simulation-based tests before testing the controller on the real plant.

\section{Acknowledgements}

The author is thankful for the support provided by the staff at VEAS wrrf, Slemmestad, Norway. 


\section{Nomenclature}

\begin{tabular}{|c|c|l|}
\hline Symbol & [Unit] & Description \\
\hline \hline$A$ & {$[\mathrm{~m} 2]$} & Surface area of liquid \\
\hline$C_{1}, C_{2}$ & - & Cost coefficients of MPC \\
\hline$e$ & - & Control error \\
\hline$F_{\text {in }}$ & {$[\mathrm{L} / \mathrm{s}]$} & Total inflow to the basin \\
\hline$F_{\text {in }_{\text {meas }}}$ & {$[\mathrm{L} / \mathrm{s}]$} & Total measured inflows to the basin \\
\hline$F_{\text {in }_{\text {nonmeas }}}$ & {$[\mathrm{L} / \mathrm{s}]$} & Total unmeasured inflows to the basin \\
\hline$F_{\text {out }}$ & {$[\mathrm{L} / \mathrm{s}]$} & Total outflow of the basin \\
\hline$F_{\text {pump }}$ & {$[\mathrm{L} / \mathrm{s}]$} & Total basin pump flow \\
\hline$h$ & {$[\mathrm{~m}]$} & Water level of basin \\
\hline$h_{\mathrm{sp}}$ & {$[\mathrm{m}]$} & Setpoint of water level of basin \\
\hline$K_{c}$ & {$[(\mathrm{~L} / \mathrm{s}) / \mathrm{m}]$} & Gain of PI controller \\
\hline$K_{i}$ & - & Process integral gain \\
\hline$N_{p}$ & {$[1]$} & Number of MPC control signal blocks \\
\hline$T_{p}$ & {$[\mathrm{~s}]$} & Prediction horizon of MPC \\
\hline$t_{0}$ & {$[\mathrm{~s}]$} & Present point of time of MPC \\
\hline$T_{c}$ & {$[\mathrm{~s}]$} & Closed-loop time-constant (Skogestad) \\
\hline$T_{\text {delay }}$ pump & {$[\mathrm{s}]$} & Approximate time-delay of basin pump \\
\hline$T_{i}$ & {$[\mathrm{~s}]$} & Integral time of PI controller \\
\hline$u$ & {$[\mathrm{~L} / \mathrm{s}]$} & Control signal to the pump \\
\hline$|\dot{u}|$ & {$[(\mathrm{L} / \mathrm{s}) / \mathrm{min}]$} & Rate of change of pump control signal \\
\hline
\end{tabular}

\section{Abbreviations}

\begin{tabular}{|l|l|}
\hline Abbr. & Full name \\
\hline \hline EKF & Extended Kalman Filter \\
\hline FT & Flow Transmitter (sensor) \\
\hline LC & Level Controller \\
\hline LT & Level Transmitter (sensor) \\
\hline MPC & Model-Predictive Control \\
\hline PID & Proportional + Integral + Derivative \\
\hline wrrf & water resource recovery facility \\
\hline
\end{tabular}

\section{References}

J. Bolmstedt. Controlling the Influent Load to Wastewater Treatment Plants. Lic. thesis, Lund University, Sweden, 2004.

L. Grüne and J. Pannek. Nonlinear Model Predictive Control. Springer-Verlag, London, 2011. doi:10.1007/978-0-85729501-9.

F. Haugen and B. Lie. Relaxed Ziegler-Nichols Closed Loop Tuning of PI Controllers. Modeling, Identification and Control, 34(2):83-97, 2013. doi:10.4173/mic.2013.2.4.

D. E. Seborg, Th. F. Edgar, and D. A. Mellichamp. Process Dynamics and Control. John Wiley and Sons, 2004.

D. Simon. Optimal State Estimation. Wiley, 2006. doi:10.1002/0470045345.

S. Skogestad. Simple analytic rules for model reduction and PID controller tuning. Journal of Process Control, 14, 2003. doi:10.1016/S0959-1524(02)00062-8.

P. J. van Overloop, R. R. Negenborn, B. De Schutter B, and N. C. van de Giesen. Predictive control for national water flow optimization in the netherlands. Intelligent Systems, Control and Automation, Springer, 42, 2010. 